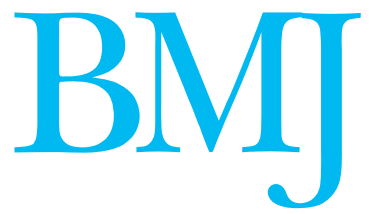

\title{
Whole body magnetic resonance imaging
}

\author{
A valuable adjunct to clinical examination
}

$\mathrm{W}$

hen Lauterbur and Damadian described the application of magnetic resonance imaging (MRI) as a clinical imaging tool in the early 1970 s the popular belief was that the technique would become the ultimate screening tool for the whole body. ${ }^{12}$ However, similar to other modalities limited by cost, acquisition times, availability, and artefact produced by motion, it evolved as a technique to image stationary body parts. Supported by technical developments in the past decade, improved excitatory pulse sequences, and faster methods of localising derived signal, and by increasing awareness of the hazards of radiation imposed by traditional techniques, the ability to use MRI as a rapid imaging tool for the whole body has now been revisited..$^{3-11}$ Reduced acquisition times have been mirrored by a logical reduction in acquisition costs, and the recent development of the moving MRI table top has facilitated the clinical introduction of this technique as a practical diagnostic tool.

\section{Oncological applications}

The principal application of whole body MRI is in detecting skeletal metastases as an alternative to skeletal

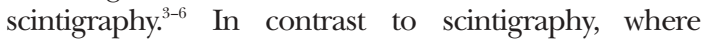
localisation of tumour deposits is indirect and requires tumour induced activity in osteoblasts, the abundance of protons in the matrix of the tumour allows direct visualisation at MRI. Regional MRI has been shown to be more sensitive than skeletal scintigraphy in the detection of skeletal metastases. More recent studies comparing whole body MRI with scintigraphy have reproduced these results, and again MRI has been found to be at least as effective as scintigraphy (fig 1 and fig 2 )..$^{3-5}$ Whole body MRI tends to better detect lesions in the spine and pelvis. In one study authors evaluating the use of whole body MRI as an alternative to bone scan reported finding metastases in 57 of 175 sites in 25 patients compared with 43 of 175 sites in the same 25 patients at scintigraphy $(\mathrm{P}<0.001) .{ }^{4}$ In a more recent study whole body MRI showed 53 of 60 metastases identified at bone scintigraphy. Although lesions in the ribs and skull were missed at magnetic resonance imaging, additional bone metastases were identified when whole body MRI was used in the spine, pelvis, and femur. ${ }^{5}$

In addition to improving detection of skeletal metastases, whole body MRI may allow simultaneous evaluation of soft tissue organs and in so doing facilitate an overview of total tumour burden in an affected patient. In effect, rather than multimodality staging integrating skeletal scintigraphy with com- puted tomography of the chest, abdomen, and pelvis at the expense of radiation dose, a single whole body MRI scan may facilitate assessment of total tumour burden, particularly in patients whose tumours spread preferentially to brain, bone, and liver, such as breast and lung tumours. In this setting, tumour staging is done without exposing the patient to radiation. In a preliminary study of 17 patients with breast carcinoma, whole body MRI allowed detection of skeletal metastases in 11 , liver metastases in five, and intracranial metastases in three patients. Interestingly, contrast enhanced computed tomography identified liver metastases in only three of the 17 patients.?

Of the patients who present with skeletal metastatic disease $15 \%$ have no known primary tumour. ${ }^{8}$ In these patients, despite an extensive search integrating serological tests, endoscopy, and imaging, a primary tumour is likely to be found in only one in five patients at a mean cost of $\$ 16000(£ 7000 ; € 12000){ }^{8}$ As an alternative, total morphological assessment of the body, as afforded by whole body MRI, may allow the detection of a primary tumour as often as the other described, costly approaches. In a preliminary study using whole body MRI in this role, primary tumours were identified in the thyroid, prostate, and lung in patients presenting with skeletal metastases predominantly in the spine. ${ }^{8}$ Similar preliminary studies have proposed a role for positron emission tomography scanning in the same group of patients, to help localise the primary tumour.

In the uncommon event of a neoplasm developing in a pregnant woman, staging information can be gained from a scan obtained by whole body MRI without the ionising risk to the fetus that is incurred by both

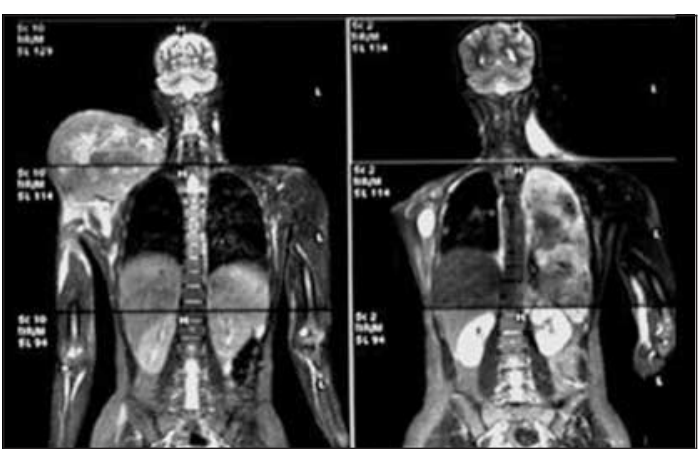

Fig 2 Whole body MR images (see bmj.com for full image) show a soft tissue sarcoma over the right shoulder at presentation (left), with axillary node, left neck, and pleural disease at 3 month follow up 
traditional approaches and positron emission tomography. ${ }^{3}$

\section{Non-oncological applications}

A worldwide fall in autopsies has prompted a search for a minimally invasive alternative. Attempting to provide an acceptable alternative, whole body MRI has been shown to be effective in the gross assessment of the corpse, helping to identify sites suitable for percutaneous biopsy, particularly in immunocompromised hosts. ${ }^{9}$ Whole body MRI has been similarly successful in whole body fat measurements and body composition research, and more recently as a primary diagnostic tool in patients with polymyositis. ${ }^{10} 11$ The advent of molecular imaging, fusing the specificity of molecular technology with the spatial resolution of imaging, is likely to herald many new scanning applications for whole body sequences. ${ }^{12}$

Stephen J Eustace consultant

(seustace@iol.ie)

\section{Erik Nelson senior registrar}

Department of Radiology, National Orthopaedic Hospital, Dublin 11, Republic of Ireland

Competing interests: The National Orthopaedic Hospital is a clinical sciences research site for Philips Medical Systems, Netherlands.

Damadian R. Tumour detection by nuclear magnetic resonance. Science 1971;171:1151.

2 Lauterbur PC. Image formation by induced interactions: examples employing nuclear magnetic resonance. Nature 1973;242:190.

3 Hargaden G, O'Connell M, Kavanagh E, Powell T, Ward R, Eustace S. Current concepts in whole body imaging using turbo short tau inversion Current concepts in whole body imaging using
recovery MR imaging. AJR 2003;180:247-52.

4 Eustace S, Tello R, Decarvalho V, et al. A comparison of whole-body turbo short tau inversion recovery MR imaging and plan technetium $99 \mathrm{~m}$ methylene diphosphonate scintigraphy in the evaluation of patients with suspected skeletal metastases. AJR 1997;169:1655-61.

5 Lauenstein TC, Freundenberg LS, Goehde SC, Ruehm SG, Goyen M, Bosk S, et al. Whole body MRI using a rolling table platform for the detection of bone metastases. Eur Radiol 2002;12:2091-9.

6 El-Khoury GY, Dalinka MK, Alazraki N, Berquist TH, Daffner RH, DeSmet AA, et al Metastatic bone disease American College of RadiolDeSmet AA, et al. Metastatic bone disease. American College of Radiol-

W. ACR Apt):S283-93. Walker R, Harper K, Eustace S. Whole body turbo STIR MR imaging in
breast carcinoma: preliminary clinical experience.J Magn Reson Imaging
$2000: 11: 343-50$.

2000;11:343-50. unknown primary tumour detection. J Magn Reson Imaging 1998;8:751-3. 9 Patriquin L, Kassarjian A, Barish M, Casserley L, O’Brien M, Andry C, et al. Postmortem whole-body magnetic resonance imaging as an adjunct to autopsy: preliminary clinical experience. J Magn Reson Imaging 2001;13:277-87.

10 Thomas EL, Saeed N, Hajnal JV, Brynes A, Goldstone AP, Frost G, et al. Magnetic resonance imaging of total body fat. J Appl Physiol Magnetic resona

11 O'Connell MI, Powell T, Brennan D, Lynch T, McCarthy C, Eustace S. Whole-body MR imaging in the diagnosis of polymyositis. AJR 2002;179:967-71.

12 Weissleder R, Mahmood U. Molecular imaging. Radiology 2001;219:316-33.

\section{Eradicating $H$ pylori}

\section{Does not increase symptoms of gastro-oesophageal reflux disease}

$\mathrm{D}$ oes eradication of Helicobacter pylori lead to an increase in symptoms of gastro-oesophageal reflux disease? We need to know this because the benefits of eradicating $H$ pylori relative to acid suppression alone are small in non-ulcer dyspepsia and uninvestigated dyspepsia. ${ }^{2}$ The absolute benefits are 6-7\%, and even a small increase in gastro-oesophageal reflux disease would be likely to make eradicating $H$ pylori in these patients of dubious benefit. New guidelines for the management of dyspepsia in Scotland and forthcoming guidelines in England and Wales recommend eradicating $H$ pylori in both non-ulcer dyspepsia and uninvestigated dyspepsia. ${ }^{34}$ In addition, advocates of population screening for and treating $H$ pylori to prevent distal gastric cancer need to ensure that the risk-benefit ratio is favourable.

The trial reported by Harvey et al in this issue does not find an increase in reflux symptoms in those randomised to eradication therapy (p 1417). ${ }^{5}$ A previous community based trial in the United Kingdom also evaluated the efficacy of $H$ pylori eradication versus placebo as part of a population "screen and treat" strategy. ${ }^{6}$ The trial reported by Harvey has the advantage of studying a wider age range and has a better response rate than this previous trial, although the prevalence of $H$ pylori was lower. A Danish study randomised subjects from the general population to an $H$ pylori screen and treat programme or to no intervention. ${ }^{7}$ These three trials show that eradicating $H$ pylori did not cause an increase in heartburn or acid regurgitation in the total of 2956 people positive for $H$ pylori who were randomised to receive antibiotics. This is consistent with a randomised controlled trial of $H$ pylori eradication in infected healthy blood donors, which did not find a statistically significant impact on reflux symptoms. ${ }^{8}$

The disadvantage of these community based trials ${ }^{567}$ is that endoscopy was not performed, so the impact of eradicating $H$ pylor $i$ in various types of upper gastrointestinal disease cannot be established. But the data are consistent with randomised controlled trials in patients having an endoscopic investigation. Eradicating $H$ pylori does not cause an increase in gastrooesophageal reflux disease in patients with peptic ulcer disease and non-ulcer dyspepsia, and no overall worsening of symptoms occurred in patients who already had reflux disease..$^{9-12 \text { w1 w2 }}$ The influence of more pathogenic strains of $H$ pylori, such as cytotoxin associated gene A positive strains, has not been well evaluated in randomised controlled trials, and eradication may exacerbate reflux disease in the rare patient with reversible achlorhydria induced by the infection. ${ }^{\text {w3 }}$ w4

Overwhelming evidence now shows that eradicating $H$ pylori has little or no impact on gastrooesophageal reflux disease in the general population. This is in contrast to a meta-analysis of observational studies that showed a negative association between $H$ pylori infection and gastro-oesophageal reflux disease. ${ }^{w 5}$ But association is not causation: improved nutrition and increased adult height may act as

Additional references w1-w6 are on bmj.com
Primary care $\mathrm{p} 1417$ 